\title{
ARTICLE OPEN \\ Saikosaponin D suppresses enterovirus A71 infection by inhibiting autophagy
}

\author{
Chang $\mathrm{Li}^{1}$, Lihong Huang ${ }^{2,3}$, Wei Sun ${ }^{2,3}$, Ying Chen ${ }^{3}$, Ming-Liang $\mathrm{He}^{2,3}$, Jianbo Yue $\mathbb{D}^{2,3}$ and Heather Ballard (iD
}

The dysregulation of autophagy, an evolutionarily conserved lysosomal degradation process, has been implicated in a wide variety of human diseases, and thus, small chemicals that modulate autophagy have therapeutic potential. Here, we assessed the ability of active components isolated from Bupleurum falcatum, a popular Chinese herb, to modulate autophagy. We found that saikosaponin $\mathrm{D}(\mathrm{SsD})$ and $\mathrm{A}(\mathrm{Ss} \mathrm{A})$ but not $\mathrm{C}(\mathrm{SsC})$ potently and reversibly inhibited the fusion of autophagosomes and lysosomes, resulting in the accumulation of autophagosomes, an increased lysosomal $\mathrm{pH}$, and TFEB nuclear translocation. RAB5A knockdown or the expression of a dominant-negative RAB5 mutant significantly reduced the ability of SsD or SsA to block autophagy. Enterovirus A71 (EV-A71), the cause of hand-foot-mouth disease, has been shown to induce autophagy. We found that SsD potently inhibited EV-A71 RNA replication and subsequent viral protein synthesis, thereby preventing EV-A71-induced cell death. ATG5 knockdown inhibited EVA71 viral protein synthesis, whereas autophagy induction by rapamycin promoted synthesis. Taken together, our data indicate that SsD and SsA are potent late-stage autophagy inhibitors that can be used to prevent EV-A71 infection.

Signal Transduction and Targeted Therapy (2019)4:4; https://doi.org/10.1038/s41392-019-0037-x

\section{INTRODUCTION}

Autophagy is an evolutionarily conserved lysosomal degradation process that is essential for maintaining the homeostasis of eukaryotic cells. ${ }^{1-3}$ Among the three types of autophagymacroautophagy, microautophagy, and chaperone-mediated autophagy - the most common and best-studied type is macroautophagy, which is hereafter referred to as "autophagy". Autophagy is initiated when cytoplasmic components, e.g. damaged organelles or misfolded proteins, are sequestered by an isolated membrane called a phagophore. After cargo is sequestered by elongating this membrane, a double-membrane vesicle called an autophagosome forms. Then, a fusion process occurs between the autophagosome and a lysosome, thereby forming an autolysosome in which the sequestered components are degraded by acidic lysosomal hydrolases. Ultimately, the digested products, such as amino acids or fatty acids, are recycled back to the cytosol to maintain cell homeostasis. ${ }^{4}$

Autophagy involvement has been shown in a wide variety of cellular processes, including cell survival, development, proliferation, and differentiation, and the dysregulation of autophagy has been implicated in various human diseases, such as cancer, neurodegeneration, and viral infection. ${ }^{5-8} \mathrm{~A}$ large number of small chemicals have been found to modulate autophagy activity, and a few have emerged as potential therapeutic drugs against autophagy-related diseases. ${ }^{9-11}$ For example, chloroquine and hydroxychloroquine have been used alone or in combination with other chemotherapy drugs in numerous clinical or preclinical trials against cancer. ${ }^{12}$ However, the majority of available autophagy drugs, including chloroquine and hydroxychloroquine, are non-specific, weakly acting, or both. ${ }^{13,14}$ Therefore, efforts to identify specific and potent autophagy modulators are necessary to identify suitable therapeutics against autophagy-related diseases.

Bupleurum falcatum is a popular Chinese herb that is commonly prescribed to treat inflammatory and infectious diseases. ${ }^{15,16}$ Triterpenoid saponins, including saikosaponin A (SsA), B (SsB), C $(\mathrm{SsC})$, and $\mathrm{D}(\mathrm{SsD})$, are active components isolated from Bupleurum falcatum. ${ }^{17}$ For example, SsD has been shown to inhibit activated T lymphocyte proliferation by downregulating the NFKB, NF-AT, and AP-1 signaling pathways. ${ }^{18}$ SsD also potently inhibits the proliferation and invasion of cancer cells, induces the apoptosis of cancer cells, and suppresses angiogenesis while exhibiting low cytotoxicity to normal human lung fibroblasts. ${ }^{19}$ In addition to the immunoregulatory and anticancer activity of saikosaponins, $\mathrm{SsD}$, SsA, and SsB have exhibited antibacterial and antiviral effects. ${ }_{i}{ }^{20}$ however, the mechanisms underlying antiviral effects remain unknown.

Enterovirus A71 (EV-A71), a positive single-stranded RNA virus, is in the Picornaviridae family. EV-A71 infection can cause diarrhea, rashes, and hand, foot and mouth disease (HFMD), with the potential development of severe neurological disease, especially among children. ${ }^{21}$ EV-A71-associated severe central nervous system disorders include polio-like acute flaccid paralysis, neurogenic pulmonary edema, and acute fatal encephalitis. ${ }^{22-26}$ However, there is currently no clinical drug against EV-A71 infection, and HFMD treatment is mostly restricted to antipyretic drugs, intravenous non-immune immunoglobulin, and glucocorticoids. $^{27}$ Although inhibitors of proteases $2 A^{\text {pro }}, 3 C^{\text {pro }}$, and $3 D$ polymerase are candidate drugs for EV-A71 infection treatment, ${ }^{28}$ no data have been provided for these treatments in preclinical trials on non-human primate models. Interestingly, it has been reported that EV-A71 infection markedly induced autophagy, and

\footnotetext{
${ }^{1}$ School of Biomedical Sciences, University of Hong Kong, Hong Kong, China; ${ }^{2}$ City University of Hong Kong ShenZhen Research Institute, ShenZhen, China and ${ }^{3}$ Department of Biomedical Sciences, City University of Hong Kong, Hong Kong, China

Correspondence: Ming-Liang He (minglihe@cityu.edu.hk) or Jianbo Yue (jianbyue@cityu.edu.hk) or Heather Ballard (ballard@hku.hk)
}

Received: 13 June 2018 Revised: 31 December 2018 Accepted: 10 January 2019

Published online: 22 February 2019 
the induced autophagy might benefit EV-A71 replication in host cells. ${ }^{29-33}$ Therefore, manipulating autophagic activity might provide an alternative therapeutic strategy against EV-A71 infections. Here, we assessed the ability of the active compounds $\mathrm{SsA}, \mathrm{SsC}$, and $\mathrm{SsD}$ isolated from Bupleurum falcatum to modulate autophagy and EV-A71 infection.

\section{MATERIALS AND METHODS}

Cell culture

HeLa and HEK293T cells were maintained in DMEM (Invitrogen, 12800-017) with 10\% FBS (Hyclone, SH30071.01) and $100 \mathrm{U} / \mathrm{ml}$ penicillin/streptomycin (Invitrogen, 15140-122) in $5 \% \mathrm{CO}_{2}$ at $37^{\circ} \mathrm{C}$. The cells were passaged every 2 days.

\section{Enterovirus-71 propagation and infection}

EV-A71 (SHZH98 strain; GenBank accession number AF302996.1) ${ }^{34-36}$ was propagated on $90-95 \%$ confluent HeLa cells in DMEM with 10\% FBS. After infection with EV-A71 for $72 \mathrm{~h}$ or when approximately $90 \%$ of the cells presented cytopathic effects, cells and media were collected. The collected samples underwent freeze-thaw cycles in liquid nitrogen. The lysates were centrifuged at $13,000 \mathrm{rpm}$ at $4{ }^{\circ} \mathrm{C}$ for $30 \mathrm{~min}$ to harvest the supernatant. EV-A71 virus was stored at $-80^{\circ} \mathrm{C}$ until use. The viral titer was detected by median tissue culture infective dose (TCID50) using the end-point dilution assay. HeLa cells were infected with EV-A71 at a multiplicity of infection (MOI) of 1.

\section{Antibodies and reagents}

The following antibodies were used: LC3 (Novus, NB100-2220), SQSTM1 (MBL, PM045), EGFR (Santa Cruz, SC-03), RAB5A (Cell Signaling Technology, 3547), LAMP1 (Cell Signaling Technology, 9091), TGN46 (BIORAD AHP500GT), EV-A71 (EMD Millipore, MAB979), GAPDH (EMD Millipore, MAB374), and dsRNA (English and Scientific Consulting, J2).

The following reagents were used: SsA (HKUST RDC, 10030), SsD (HKUST RDC, 10031), SsC (HKUST RDC, 10032), glycyl-L-phenylalanine-ß-naphthylamide (GPN, Santa Cruz, SC-252858), thapsigargin (TG, Sigma-Aldrich, T9033), bafilomycin A1 (BAF, SigmaAldrich, B1793), rapamycin (Sigma-Aldrich, R8781), Fura-2 AM (Invitrogen, F1221), LysoSensor Green DND-189 (Invitrogen, L7535), LysoSensor Yellow/Blue DND-160 (Invitrogen, L7545), RNAscope ${ }^{\circledR}$ Probe-V-EV-A71-PP (Advanced Cell Diagnostics, 489071), RNAscope ${ }^{\circledR}$ Multiplex Fluorescent Detection Kit (Advanced Cell Diagnostics, 320850), TFEB siRNA (L-050607-020005), and nontarget siRNA (Dharmacon).

Construction of shRNA expression vectors and production and infection of lentivirus

Two optimal 21-mers were selected in the human RAB5A ${ }^{37}$ and RAB7 genes. One 21-mer was selected in GFP as a control. To generate shRNA, the 21-mers were incorporated into the pLKO.1 vector. HEK293T cells were used to produce lentivirus. Briefly, HEK 293T cells were seeded at $4 \times 10^{5}$ cells/well in 6-well plates, and the medium was replaced with antibiotic-free media the next day. PLKO.1-shRNA or pLenti-CMV-DEST vectors were mixed with the lentivirus envelope and package plasmids PMD2.G (Addgene) and psPAX2 (Addgene) in Opti-MEM for the target plasmid mixture. Another mixture of lipofectamine 2000 in Opti-MEM was prepared. After incubation for $5 \mathrm{~min}$ at room temperature, these two mixtures were combined and incubated for another $30 \mathrm{~min}$. This mixture was added to the HEK $293 \mathrm{~T}$ cells. Normal media were replaced after $12 \mathrm{~h}$. The viruses were harvested twice at $36 \mathrm{~h}$ and $60 \mathrm{~h}$ after transfection. For infections, cells were seeded at $2 \times 10^{5}$ cells/well in 6-well plates. The next day, cells were infected with the targeted lentiviruses in regular medium containing $8 \mu \mathrm{g} / \mathrm{mL}$ polybrene. Cells were selected in fresh medium containing puromycin $(3 \mu \mathrm{g} / \mathrm{mL}) 48 \mathrm{~h}$ after infection. After selection for 2 days,
shRNA knockdown efficiencies were tested by Western blot analysis.

\section{Transient transfection}

Cells were plated in a 24 -well plate at $6 \times 10^{4}$ cells/well. The next day, regular medium was replaced with antibiotic-free medium before transfection. Two mixtures were prepared, one containing $0.5 \mu \mathrm{l}$ Lipofectamine 2000 in $25 \mu \mathrm{l}$ Opti-MEM for each well, and another containing $0.5 \mu \mathrm{g}$ DNA plasmids in $25 \mu \mathrm{l}$ Opti-MEM/well. After $5 \mathrm{~min}$ incubation at room temperature, these two mixtures were combined and incubated for another $30 \mathrm{~min}$. Finally, cells were transfected with the combined $50-\mu$ mixture. The medium was replaced with fresh medium $4-6 \mathrm{~h}$ after transfection. After transfection for $24-48 h$, the cells were ready for the following experiments.

Western blot analyses

Cells were washed with $1 \mathrm{x}$ phosphate-buffered saline (PBS) twice and then lysed with ice-cold EBC protein lysis buffer. The lysates were homogenized several times by 25 -gauge needles and then centrifuged at $13,200 \mathrm{rpm}$ for $15 \mathrm{~min}$ at $4{ }^{\circ} \mathrm{C}$ to remove debris. Protein concentrations were detected by the Bradford protein assay (Bio-RAD). After denaturation by $5 x$ SDS sample loading buffer at $99^{\circ} \mathrm{C}$ for $8 \mathrm{~min}, 30-50 \mu \mathrm{g}$ protein per sample was loaded in $8-15 \%$ SDS-PAGE gels according to the different molecular weights of the proteins. After electrophoresis, proteins were transferred to PVDF membrane (Millipore), and then the membranes were blocked with $5 \%$ non-fat milk in TBST $(20 \mathrm{mM}$ Tris- $\mathrm{HCl}, \mathrm{pH} 7.6,150 \mathrm{mM} \mathrm{NaCl}, 0.1 \%$ Tween-20) for $1 \mathrm{~h}$ at room temperature. The membrane was incubated with the appropriate primary antibodies overnight at $4{ }^{\circ} \mathrm{C}$. The next day, the membrane was incubated with the respective secondary antibody for $1 \mathrm{~h}$. Protein levels were detected by chemiluminescence after washing membranes several times and were normalized to GAPDH as a loading marker.

\section{Immunofluorescence analysis}

After drug treatment or EV-A71 infection, cells were fixed with 4\% paraformaldehyde (PFA) for $30 \mathrm{~min}$ at room temperature. Then, cells were blocked with blocking buffer ( $1 \%$ bovine serum albumin (BSA), 1\% donkey serum) in PBST (1x PBS, 0.01\% Tween-20 and $0.1 \%$ Triton) for $1 \mathrm{~h}$ and incubated with different primary antibodies overnight at $4{ }^{\circ} \mathrm{C}$. The next day, cells were incubated with the respective secondary fluorescent antibody for $1.5-3 \mathrm{~h}$ and mounted with Prolong Gold Antifade Reagent (Invitrogen). Images were taken using a Carl Zeiss LSM 710 confocal microscope with a $63 \times$ oil objective (NA 1.4) and analyzed with Zen 2011 software. The co-localization coefficient analysis of images was performed using the Zeiss LSM 710 Software as follows: (a) correct background settings for the images, (b) crop region of interest $(\mathrm{ROI}),(\mathrm{c})$ generate histogram of $\mathrm{ROI}$, and (d) analyze the results, e.g., co-localized area, the ratio of colocalization, and co-localization coefficient.

\section{Transmission electron microscopy (TEM)}

After drug treatment, the Thermanox tissue culture cover slip (with cells face up) was placed into a glass vial containing primary fixative $(2.5 \%$ glutaraldehyde and $2 \%$ paraformaldehyde in $0.1 \mathrm{M}$ cacodylate buffer ( $\mathrm{pH} 7.2$ ) with $0.05 \% \mathrm{CaCl}_{2}$ ) for $2 \mathrm{~h}$ at room temperature. After rinsing the cells with $0.1 \mathrm{M}$ cacodylate buffer three times for $5 \mathrm{~min}$ each, the cells were washed twice with $0.1 \mathrm{M}$ cacodylate buffer for $10 \mathrm{~min}$. Cells were then fixed with $2 \% \mathrm{OsO}_{4}$ in $0.1 \mathrm{M}$ cacodylate buffer $(\mathrm{pH}$ 7.4) with $1.5 \%$ potassium ferrocyanide in the dark for $2 \mathrm{~h}$ and were rinsed again. The cells were sequentially washed with 0.05 $\mathrm{M}$ cacodylate buffer, $0.025 \mathrm{M}$ cacodylate buffer, and $\mathrm{ddH}_{2} \mathrm{O}$ for $10 \mathrm{~min}$ each. To dehydrate the cells, $30 \%$ ethanol, $50 \%$ ethanol, $70 \%$ ethanol, $80 \%$ ethanol, $90 \%$ ethanol, $95 \%$ ethanol, $100 \%$ 
ethanol, $3: 1 \quad 100 \%$ ethanol:100\% acetone, $1: 1 \quad 100 \%$ ethanol:100\% acetone, 1:3 100\% ethanol:100\% acetone, and 100\% acetone were used in order. Subsequently, cells were infiltrated using 3:1 100\% acetone:Spurr's resin, 1:1 100\% acetone:Spurr's resin, 1:3 100\% acetone:Spurr's resin, and Pure Spurr's resin with rotation. Next, cells were embedded in plastic mold for half a day at room temperature and then baked in an oven at $70^{\circ} \mathrm{C}$ for 2 days. Ultrathin sections (approximately $90 \mathrm{~nm}$ ) were cut and stained with $8 \%$ uranylacetate and lead citrate prior to image collection under TEM.

MTT cell viability assay

Cells were cultured in 96-well plates at $1 \times 10^{4}$ cells/well. After pretreatment with SsD and EV-A71 infection for 2-4 days, cells were incubated with $10 \%(\mathrm{v} / \mathrm{v}) \mathrm{MTT}$ solution (USB Corporation, 19265) in regular medium for $3 \mathrm{~h}$ before removing solution. Immediately, $150 \mu \mathrm{L}$ of DMSO solution was added to each well, and the absorbance of the resulting purple formazan solution was measured at $570 \mathrm{~nm}$ with a reference wavelength of $630 \mathrm{~nm}$ on a microplate reader (Techan infinite M200).

\section{Measurement of lysosomal $\mathrm{pH}$}

Cells were incubated with $1 \mu \mathrm{M}$ LysoSensor Green DND-189, a fluorescent probe for qualitatively detecting the $\mathrm{pH}$ of acidic organelles, in fresh medium for $20 \mathrm{~min}$ at $37^{\circ} \mathrm{C}$. Fluorescence was analyzed immediately using a microplate reader with excitation and emission set at 485 and $530 \mathrm{~nm}$, respectively.

In addition, a pH calibration curve was generated according to a previous protocol. ${ }^{38}$ Briefly, cells were plated in a 96-well plate. The next day, cells were incubated with Lysosensor Yellow/Blue DND-160 (2 $\mu \mathrm{M})$, a ratiometric lysosomal pH dye for quantitatively determining the lysosomal $\mathrm{pH}$, for $30 \mathrm{~min}$ at $37^{\circ} \mathrm{C}$ in fresh medium, and cells were then treated with $10 \mu \mathrm{M}$ monensin (Sigma, M5273) and $10 \mathrm{M}$ nigericin (Sigma, N7143) in $25 \mathrm{mM}$ 2-(N-morpholino) ethanesulfonic acid (MES) calibration buffer ( $5 \mathrm{mM} \mathrm{NaCl}, 115 \mathrm{mM} \mathrm{KCl}$ and $1.2 \mathrm{mM} \mathrm{MgSO}_{4}, \mathrm{pH} 3.5-6.0$ ) for $10 \mathrm{~min}$. Fluorescence was detected in a microplate reader (excitation/emission $=340 / 440$ and $384 / 540 \mathrm{~nm}$ ). The ratio of fluorescence absorbance with 340 and $380 \mathrm{~nm}$ excitation was plotted against the $\mathrm{pH}$ values in MES buffer using Microsoft Excel software.

RNA in situ hybridization

To visualize the EV-A71 genome, in situ RNA hybridization was performed. ${ }^{39}$ Cells were seeded on cover glasses in 48-well plates at $3 \times 10^{4}$ cells/well. After drug treatments and EV-A71 infection, cells were fixed with $10 \%$ neutral buffered formalin (NBF) for $30 \mathrm{~min}$ at room temperature. The cells were washed with 1x PBS twice, and then diluted protease $(1: 15)$ was added to cells with $1 \times$ PBS to release RNA in the cells. After incubation with diluted protease for $10 \mathrm{~min}$, cells were hybridized with the target probe for $2 \mathrm{~h}$ at $40^{\circ} \mathrm{C}$ and then sequentially with Amp 1FL (30 min), Amp 2-FL (15 min), Amp 3-FL (30 min), and Amp 4$\mathrm{FL}(15 \mathrm{~min})$ at $40^{\circ} \mathrm{C}$ to amplify the RNA signal. Before each amplification process, the cells were washed twice with $1 \times$ wash buffer. After incubation with blocking buffer for $1 \mathrm{~h}$, experiments were performed as described above in the immunofluorescence analysis section.

Quantification of intracellular and extracellular viral genomic RNA HeLa cells were pretreated with SsD for $4 \mathrm{~h}$, washed twice with $1 \times$ PBS, and infected with EV-A71 at an MOI of 1. EV-A71 virus was allowed to be internalized into cells for $1 \mathrm{~h}$. Unattached viruses were washed out twice with PBS, and $200 \mu \mathrm{l}$ regular medium containing $2 \%$ FBS was added to each well. Total RNA was isolated from infected cells or culture medium to measure the intracellular viral RNA or extracellular viral RNA in the virions at different time points, and qRT-PCR assays were performed. ${ }^{34}$
Virus titration assay

HeLa cells were pretreated with SsD for $4 \mathrm{~h}$ and then washed twice with $1 \times$ PBS. Ten-fold dilutions of EV-A71 stock were prepared before EV-A71 infection. EV-A71 virus was allowed to be internalized into cells for $1 \mathrm{~h}$. After incubation for $1 \mathrm{~h}$, cells were covered with agar, which formed a gel. A circular zone-like plaque was formed by each infectious particle. To stain living cells and distinguish between living cells and plaques, crystal violet was added to cells. The titer of EV-A71 was quantified as plaqueforming units (PFU) per milliliter and calculated by counting plaques. Each dilution was plated in triplicate to ensure accuracy.

Intracellular $\mathrm{Ca}^{2+}$ measurement

Cells were seeded at $7 \times 10^{4}$ cells per well in a 24 -well plate. The next day, cells were pretreated with SsD for $6 \mathrm{~h}$ and then incubated with Fura-2 AM $(4 \mu \mathrm{M})$ in regular HBSS (Invitrogen, 14175-059) at room temperature for 20-30 min in the dark. Subsequently, cells were washed three times with calcium-free HBSS containing $2 \mathrm{mM}$ EGTA (Sigma, 3889). Finally, cells were placed on the stage of an Olympus inverted epifluorescence microscope with a $\times 20$ objective and challenged with $200 \mu \mathrm{M}$ GPN or $1 \mu \mathrm{M}$ TG at room temperature for $10 \mathrm{~min}$. Fluorescence images were collected by excitation alternating between $340 \mathrm{~nm}$ and 380 $\mathrm{nm}$ with emission at $510 \mathrm{~nm}$. Images were captured every $5 \mathrm{~s}$ and processed by Cell $R$ imaging software.

Statistical analysis

Data are presented as the means \pm S.E.M. The statistical significance of differences was determined by one-way ANOVA or Student's $t$ test. $P<0.05$ was regarded as significant.

\section{RESULTS}

Ss $D$ and $S s A$ inhibit autophagosome-lysosome fusion in HeLa cells Triterpenoid saponins, e.g., SsA, SsC, and SsD, are the active compounds in Bupleurum falcatum (Fig. 1a). We first assessed the ability of these three saponins to modulate autophagy in RFP and GFP tandem-tagged LC3-expressing HeLa cells. As shown in Fig. $1 \mathrm{~b}$, both $\mathrm{SsD}$ and $\mathrm{SsA}$, but not $\mathrm{SsC}$, markedly induced yellow LC3-II puncta formation in HeLa cells, whereas almost no red-only LC3-II puncta were present in SsD-treated or SsA-treated cells. These data suggested that the fusion between autophagosomes and lysosomes is blocked in SsD-treated or SsA-treated cells. Likewise, the treatment of cells with SsD or SsA, but not SsC, markedly increased the levels of both LC3-II and p62, a cargo receptor for the autophagic degradation of ubiquitinated proteins, in both time-dependent and concentration-dependent manners (Fig. 1c-f), which also suggested the inhibition of autophagosomal-lysosomal fusion in SsA-treated or SsD-treated cells. Interestingly, we also found that the accumulated LC3-II and p62 (SQSTM1) in SsD-treated cells was reduced after washing out $\mathrm{SsD}$, indicating that the effects of SsD on autophagy are reversible (Fig. 1g). We further obtained transmission electron microscope images of HeLa cells treated with SsD or SsA. As shown in Fig. 2a, SsD or SsA treatment of cells significantly increased the number of autophagosome-like structures compared to control cells. Because SsD and SsA are epimers with very similar effects on autophagy, we primarily studied SsD in the subsequent studies.

To confirm that SsD inhibits the fusion between autophagosomes and lysosomes, we treated control or SsD pre-treated HeLa cells with bafilomycin A1 (BAF), a vacuolar proton ATPase inhibitor that can arrest autophagosomal-lysosomal fusion in cells. As shown in Fig. 2b, BAF treatment did not induce further accumulation of LC3-II and p62 in SsD-treated HeLa cells compared to control cells. Similar results were observed in SsAtreated cells (Fig. S1). These data suggested that SsD and SsA, like $\mathrm{BAF}$, inhibit autophagosome-lysosome fusion, thereby resulting in the accumulation of both LC3-II and p62. To further confirm that 
A

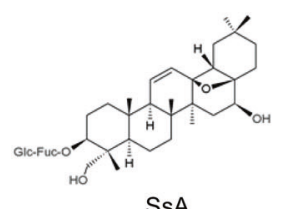

SsA

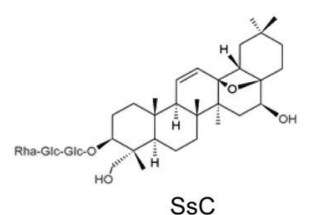

$\mathrm{SsC}$

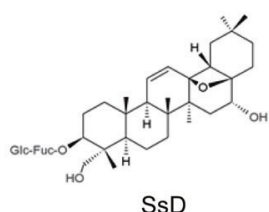

SsD

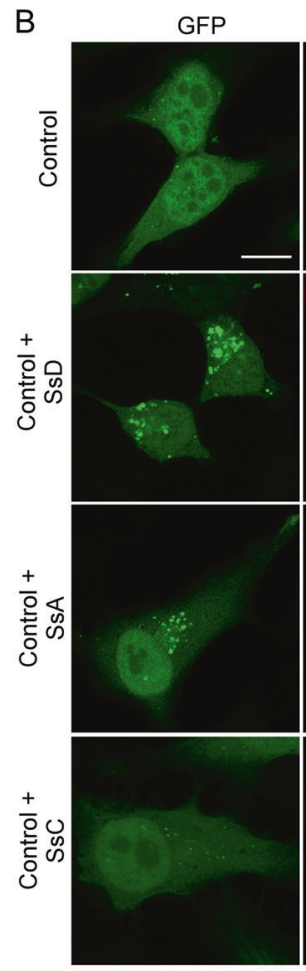

RFP
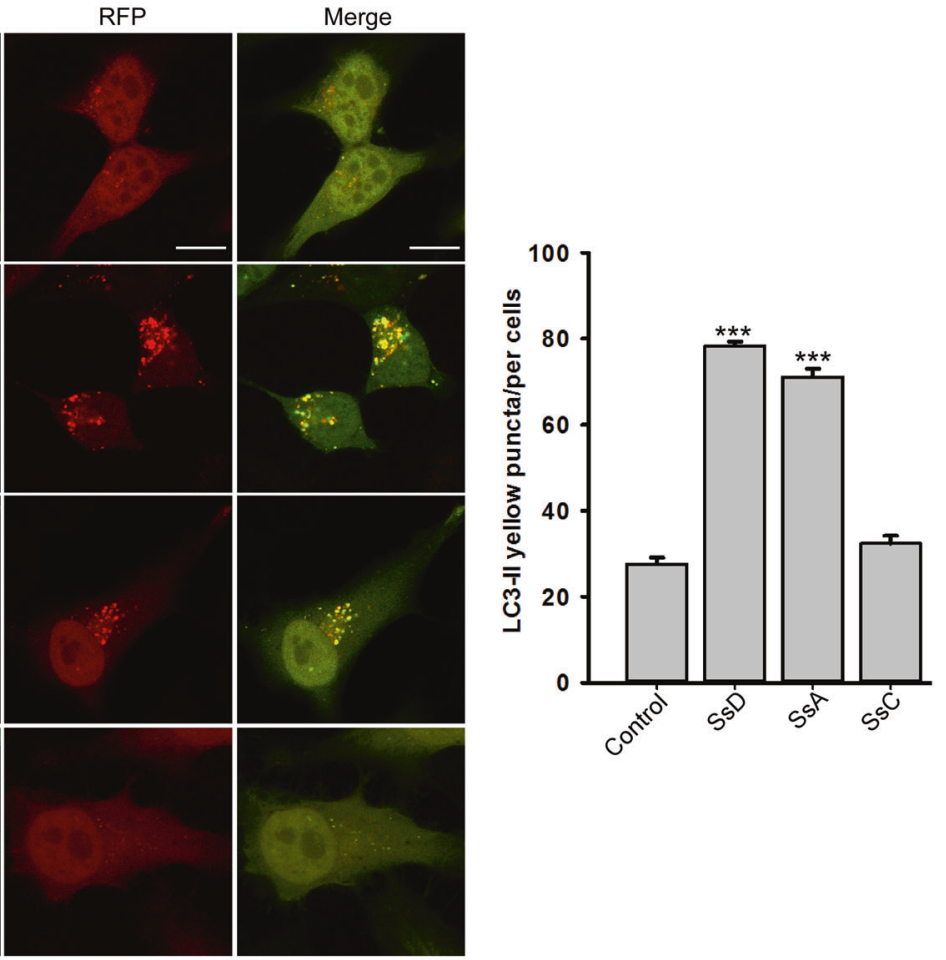

C

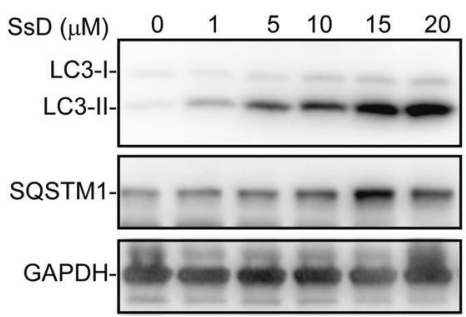

$\mathrm{F}$

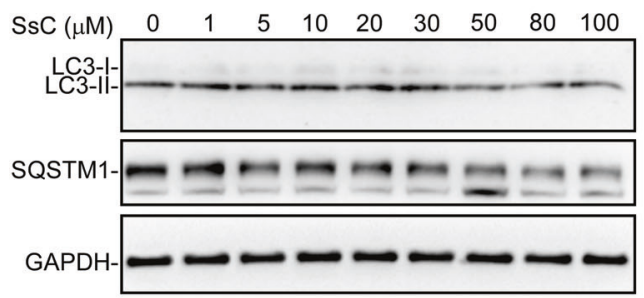

D

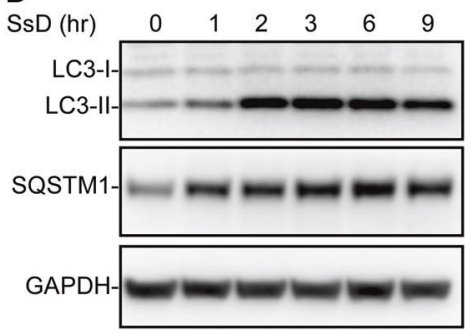

E

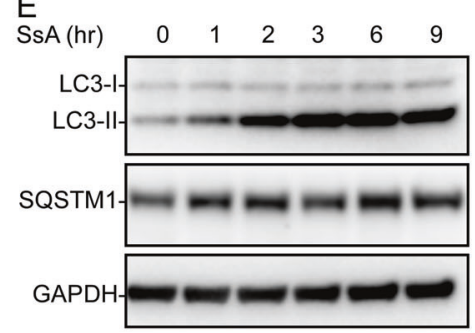

SsD 6h and

G

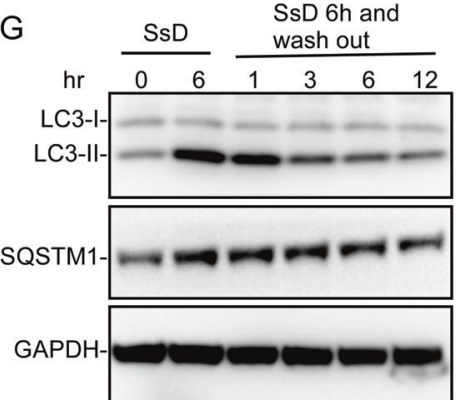

Fig. 1 SsD and SsA inhibit autophagy in HeLa cells. a Structures of SsA, SsC, and SsD. b SsD (15 $\mu$ M) and SsA ( $30 \mu M)$, but not SsC, significantly increased LC3-II yellow puncta formation but did not significantly affect LC3-II red-only puncta in RFP-GFP-LC3-expressing HeLa cells. Scale bar $=10 \mu \mathrm{m}$. Quantification of LC3 yellow puncta/red puncta (\%) is presented as the mean \pm S.E., $n=\sim 80$ cells from 3 independent experiments. c Treatment of HeLa cells with SSD for $6 \mathrm{~h}$ induced the accumulation of both LC3-II and SQSTM1 in a dose-dependent manner. d SsD $(15 \mu \mathrm{M})$ induced the accumulation of both LC3-II and SQSTM1 in HeLa cells in a time-dependent manner. e SsA (30 $\mu$ M) induced the accumulation of both LC3-II and SQSTM1 in HeLa cells in a time-dependent manner. $\mathbf{f}$ Treatment with different concentrations of SsC for $6 \mathrm{~h}$ failed to induce the accumulation of either LC3-II or SQSTM1 in HeLa cells. g SsD $(15 \mu \mathrm{M})$ reversibly inhibited autophagy 

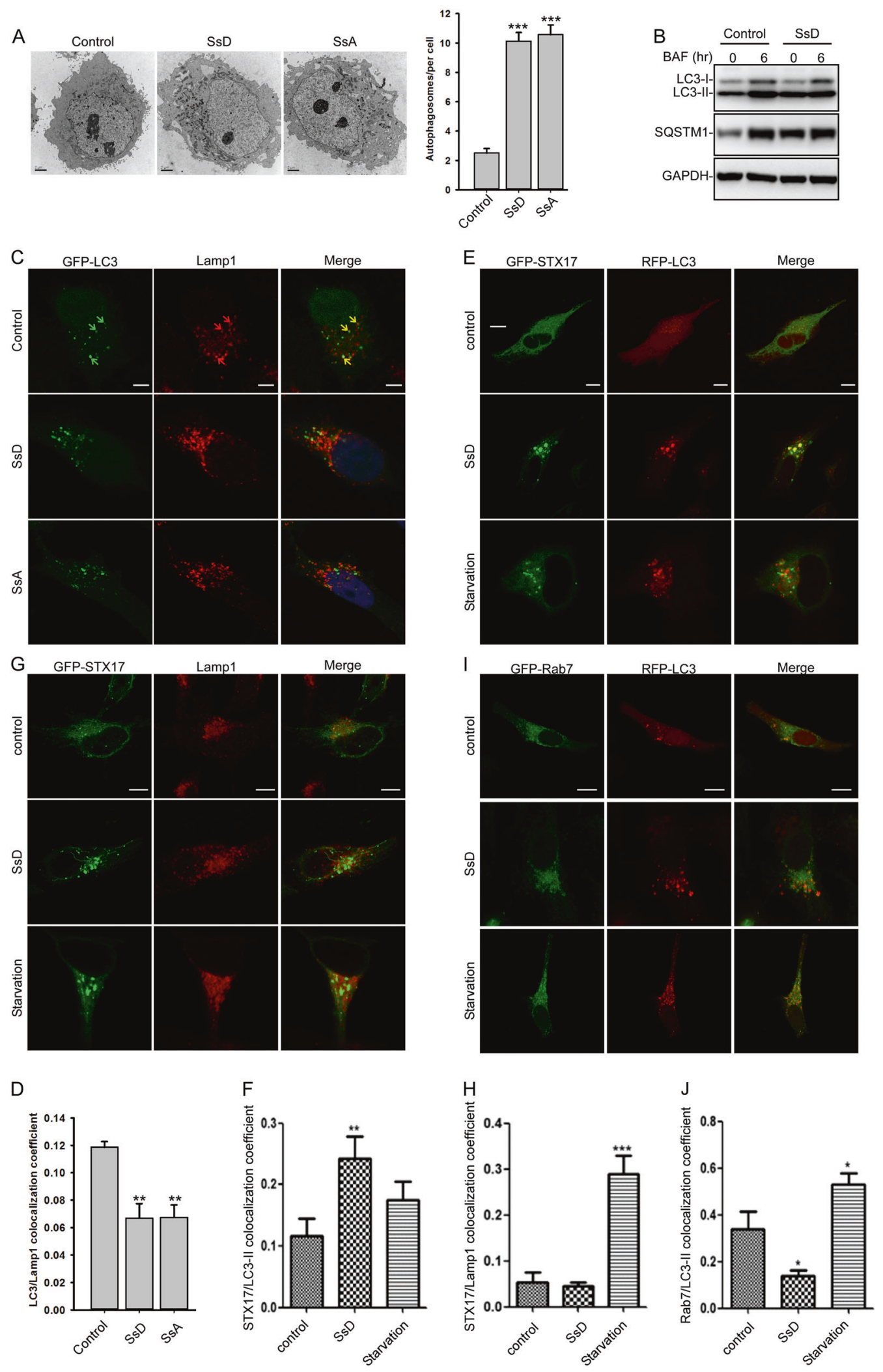

the SsD-induced LC3-II puncta represent autophagosomes, we assessed the co-localization of LC3-II, Lamp1 (a lysosomal marker), STX17 (a SNARE protein locating at the autophagosome), and RAB7 (a small GTPase located at late endosome or lysosome) in SsD-treated cells. SsD-induced or SsA-induced LC3-II puncta

exhibited weak co-localization with Lamp1 (Fig. 2c, d). However, SsD-induced LC3-II puncta were almost completely co-localized with STX17 puncta (Fig. 2e, f). Likewise, SsD-induced STX17 puncta exhibited weak co-localization with Lamp1, whereas starvation significantly induced their co-localization (Fig. 2g, h). Moreover, 
Fig. 2 SsD induces the accumulation of autophagosomes. a SsD $(15 \mu \mathrm{M})$ and SsA $(30 \mu \mathrm{M})$ induced the accumulation of autophagic vacuoles as shown in the electron micrographs. Quantification of autophagosomes per cell is presented as the mean \pm S.E., $n=\sim 20$ to 40 cells. The asterisks $\left(^{*}\right)$ symbols indicate $p<0.05$ by $t$ test analysis. b BAF $(100 \mathrm{nM})$ treatment for $6 \mathrm{~h}$ failed to further induce the accumulation of either LC3-II or SQSTM1 in control or SsD $(15 \mu \mathrm{M})$-treated HeLa cells. c, d HeLa cells were transfected with GFP-LC3 and treated with or without SsD $(15 \mu \mathrm{M})$ or SsA $(30 \mu \mathrm{M})$ before Lamp1 immunostaining and confocal imaging (c). The quantification of LC3-II/Lamp1 co-localization is presented as the mean \pm S.E., $n=\sim 80$ to 100 cells of 3 independent experiments (d). e, f HeLa cells were transfected with GFP-STX17 and RFPLC3 and treated with or without SsD $(15 \mu \mathrm{M})$ or underwent starvation followed by confocal imaging (e). The quantification of LC3-II/STX17 colocalization is expressed as the mean \pm S.E., $n=3$ (totally 15-30 cells) (f). g, h HeLa cells were transfected with GFP-STX17 and treated with or without SsD $(15 \mu \mathrm{M})$ or underwent starvation prior to Lamp1 immunostaining and confocal imaging (g). The quantification of STX17/Lamp1 co-localization is expressed as the mean \pm S.E., $n=3$ (totally 15-30 cells) (h). $\mathbf{i}$, $\mathbf{j}$ HeLa cells were transfected with GFP-RAB7 and RFP-LC3 and treated with or without SsD $(15 \mu \mathrm{M})$ or underwent starvation prior to confocal imaging (i). The quantification of RAB7/LC3-II co-localization is expressed as the mean \pm S.E., $n=3$ (totally $15-30$ cells) (j). Scale bar $=10 \mu \mathrm{m}$. Differences between control group and treatment groups were analyzed by one-way ANOVA

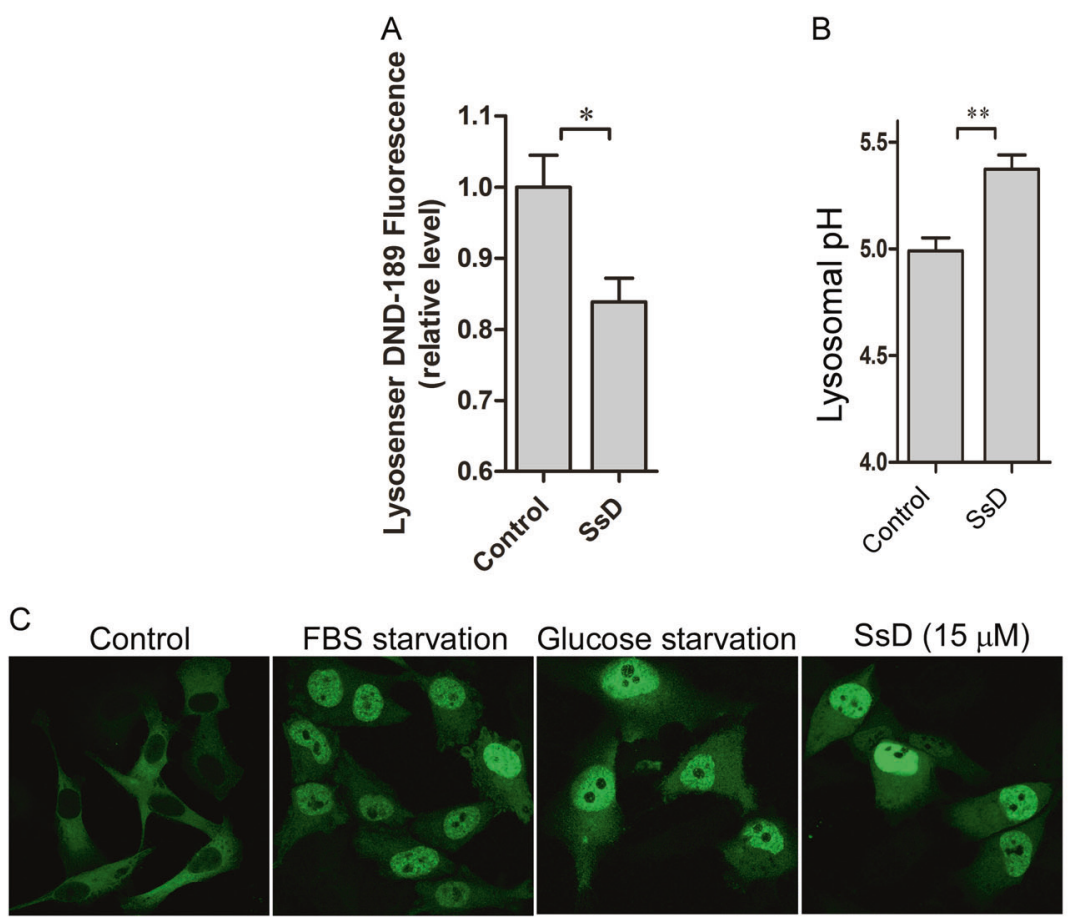

TFEB-GFP

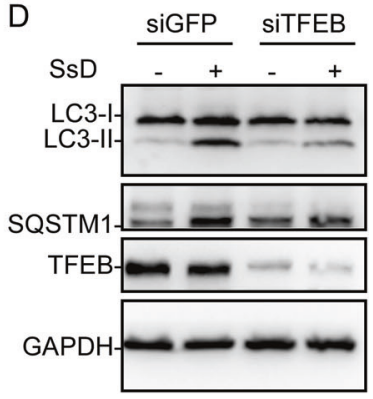

Fig. $3 \mathrm{SsD}$ treatment of HeLa cells increases lysosomal $\mathrm{pH}$ and induces TFEB nuclear translocation. $\mathbf{a}$, $\mathbf{b}$ SsD (15 $\mu \mathrm{M})$ led to an increase of lysosomal pH in HeLa cells as measured using Lysosensor DND-189 (a) or the quantitative ratiometric LysoSensor Yellow/Blue DND-160 (b). The graphs in (b) and (c) represent data from three independent experiments, and data are expressed as the means \pm S.D., $n=3$. The asterisk (*) symbols indicates $p<0.05$ by $t$-test analysis. c HeLa cells were transfected with TFEB-GFP and treated with or without SsD (15 $\mu \mathrm{M})$ or underwent starvation before confocal imaging. d HeLa cells were transfected with siGFP or siTFEB and treated with or without SsD (15 $\mu \mathrm{M}$ ), and then LC3, p62, TFEB, or GAPDH immunoblot analyses were performed

SsD failed to induce the co-localization between LC3-II and RAB7, whereas starvation significantly induced co-localization (Fig. 2d). Taken together, these data demonstrated that the SsD-induced LC3-II puncta represented autophagosomes, not autolysosomes. Therefore, our results indicated that SsD and SsA, like BAF, inhibit the fusion between autophagosomes and lysosomes, resulting in the accumulation of autophagosomes and increased levels of LC3II and p62.

SsD alkalizes lysosomal $\mathrm{pH}$ and induces TFEB nuclear translocation in HeLa cells

Because acidic lysosomal $\mathrm{pH}$ is essential for autophagosomelysosome fusion, ${ }^{40}$ we next examined whether SsD treatment of cells changes lysosomal $\mathrm{pH}$. Cells were labeled with LysoSensor Green DND-189 (pKa $=\sim 5.2){ }^{41}$ its fluorescence intensity is decreased when $\mathrm{pH}$ is increased. As shown in Fig. 3a, SsD treatment significantly decreased the fluorescence intensity of lysosomes, suggesting that lysosomal $\mathrm{pH}$ is increased in SsD- treated cells. Furthermore, lysosomal $\mathrm{pH}$ was quantified by the quantitative ratiometric LysoSensor Yellow/Blue DND-160 42 assay. As shown in Fig. 3b, lysosomal pH was increased from pH 4.99 in control cells to $\mathrm{pH} 5.37$ in SsD-treated cells. Similar results were observed in SsA-treated cells (Fig. S2). Therefore, these data indicated that SsD and SsA, again with similarities to BAF and chloroquine, alkalizes lysosomal $\mathrm{pH}$, which likely contributes to the autophagosome-lysosome fusion blockage.

It has been shown previously that TFEB, a transcription factor important for autophagy progression, is associated with lysosomes when cells are maintained in normal medium due to its phosphorylation by mTOR, whereas starvation triggers the nuclear localization of dephosphorylated TFEB because of the inactivation of mTOR. $^{43,44}$ We therefore examined whether SsD treatment alters TFEB lysosomal localization. As shown in Fig. $3 \mathrm{C}$ and S3A, SsD treatment, similarly to FBS or glucose starvation, markedly induced the nuclear localization of TFEB. However, SsD treatment did not affect mTOR activation (Fig. S3B), suggesting that 
A
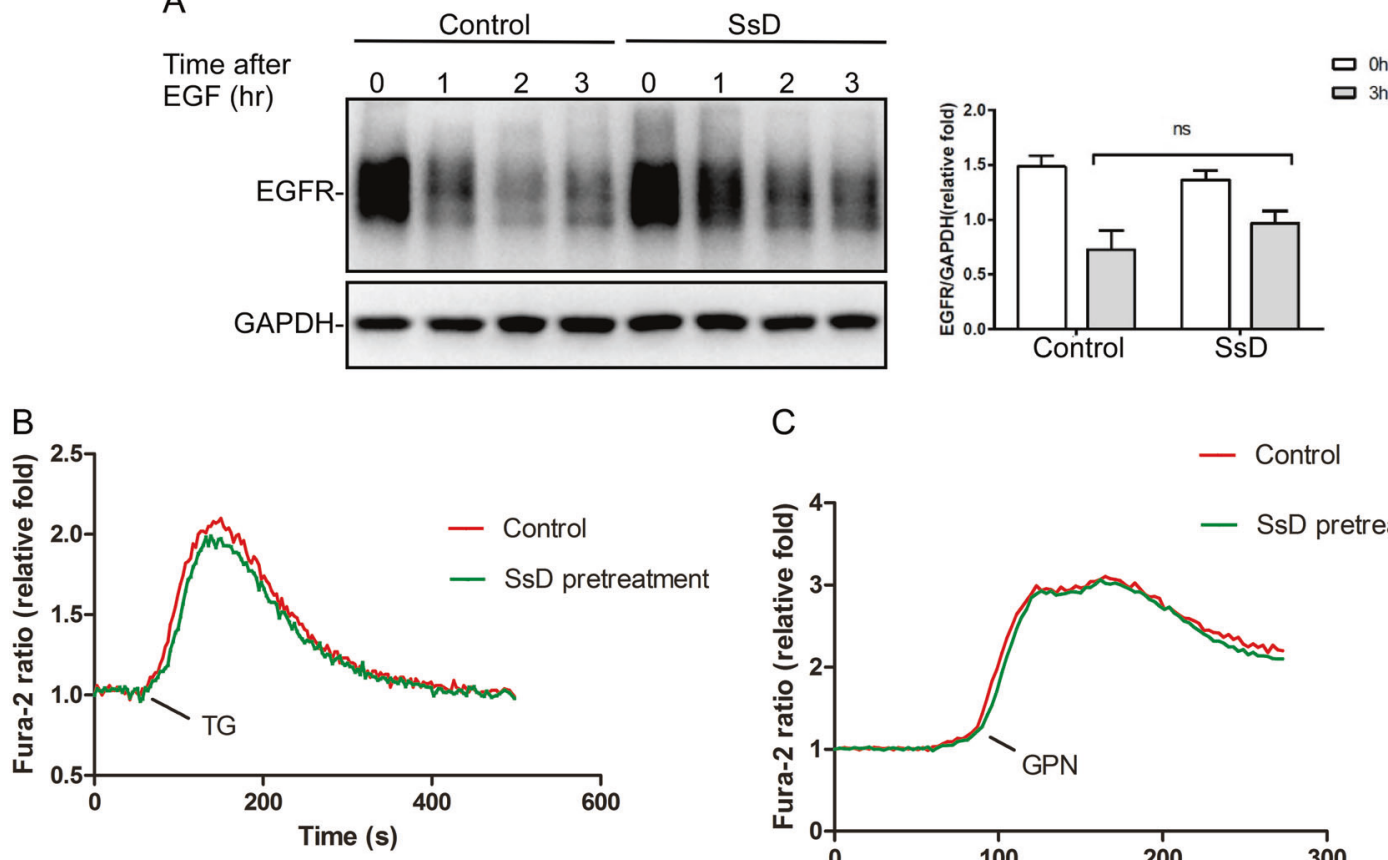

C
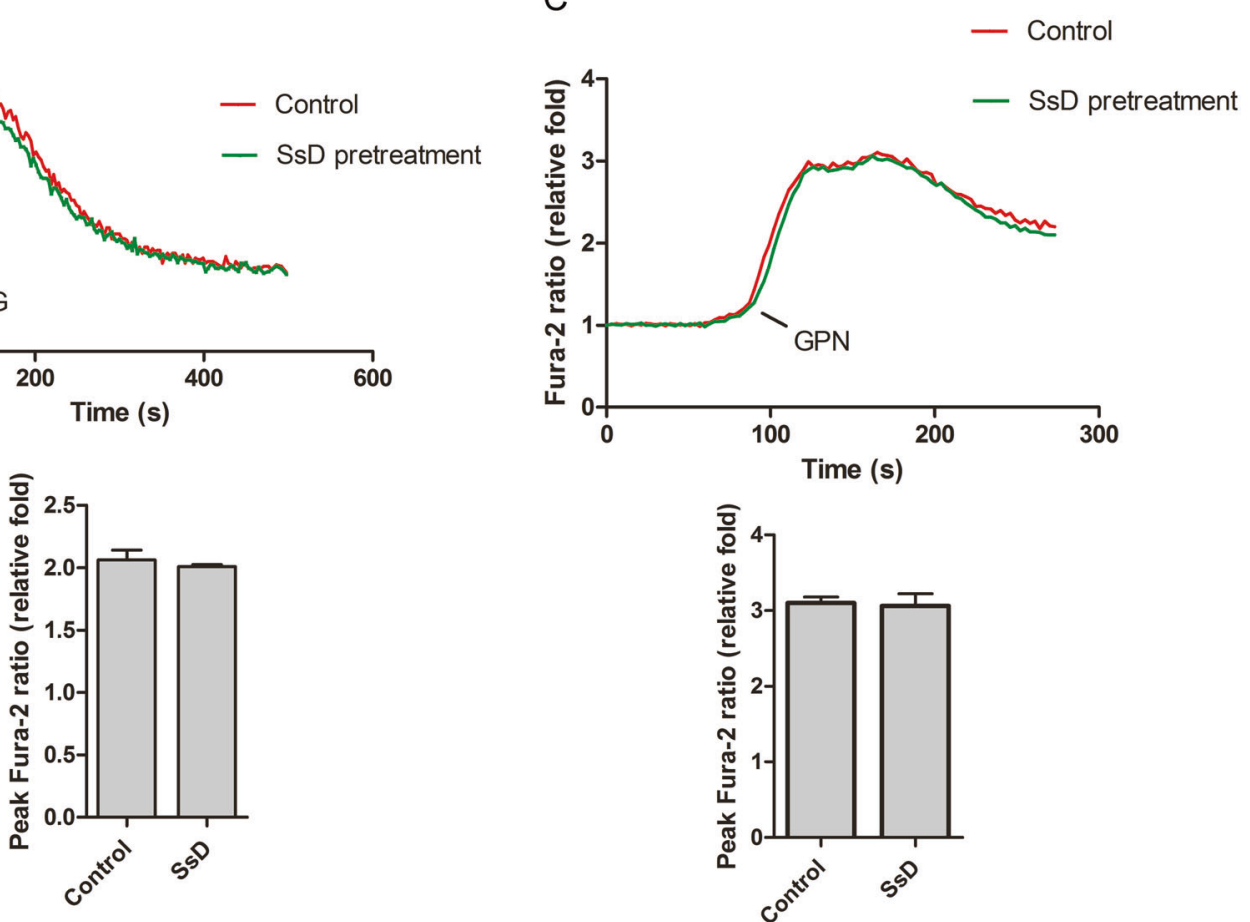

Fig. $4 \mathrm{SsD}$ delays the endocytosis pathway in HeLa cells and does not alter $\mathrm{Ca}^{2+}$ content in the lysosome or ER. a HeLa cells were treated with EGF in the presence or absence of SsD $(15 \mu \mathrm{M})$ and subjected to EGFR immunoblot analyses. $\mathbf{b}$ SsD $(15 \mu \mathrm{M})$ pretreatment for $6 \mathrm{~h}$ failed to affect the ability of TG $(1 \mu \mathrm{M})$ to release ER Ca ${ }^{2+}$ in Fura-2-loaded HeLa cells in the absence of extracellular Ca ${ }^{2+}$. c SsD $(15 \mu \mathrm{M})$ pretreatment for $6 \mathrm{~h}$ did not affect the ability of GPN $(200 \mu \mathrm{M})$ to release lysosomal $\mathrm{Ca}^{2+}$ in Fura-2-loaded HeLa cells in the absence of extracellular Ca ${ }^{2+}$. The graphs in (b) and (c) represent data from three independent experiments, and data are expressed as the means \pm S.D., $n=3$. The * symbols indicate $p<0.05$ by $t$-test analysis

SsD-induced TFEB nuclear localization is mTOR independent. As expected, TFEB knockdown abolished SsD-induced LC3-II and p62 accumulation (Fig. 3d), which is in agreement with the general role of TFEB in autophagy progression and lysosome biogenesis. $^{44,45}$

SsD delays the endocytosis pathway in HeLa cells and does not alter $\mathrm{Ca}^{2+}$ content in lysosomes or the ER

Because the lysosomal pH was increased in SsD-treated and SsAtreated cells, we performed EGFR (epidermal growth factor receptor) degradation assays to assess whether SsD affects the general endosomal-lysosomal pathway. HeLa cells were pretreated with or without SsD before EGF addition. EGF binding to its receptor (EGFR) triggered EGFR translocation from the plasma membrane to the lysosome to be degraded; however, SsD pretreatment only delayed but did not block the degradation of EGFR (Fig. 4a), suggesting that SsD has marginal effects on general endosomal-lysosomal degradation.

Because intracellular $\mathrm{Ca}^{2+}$ is important for autophagy progression and membrane fusion ${ }^{46-49}$ and because it was previously suggested that SsD could inhibit sarco/endoplasmic reticulum $\mathrm{Ca}^{2+}$
ATPase (SERCA) in the ER to trigger $\mathrm{ER} \mathrm{Ca}^{2+}$ release, we assessed the effects of SSD on ER $\mathrm{Ca}^{2+}$ content. As shown in Fig. 4b, thapsigargin (TG), an inhibitor of SERCA, triggered increases in cytosolic $\mathrm{Ca}^{2+}$ to similar levels in the presence or absence of SsD pretreatment, indicating that $\mathrm{ER} \mathrm{Ca}^{2+}$ pools are not affected by SsD treatment. Lysosomes are also intracellular $\mathrm{Ca}^{2+}$ pools, and $\mathrm{Ca}^{2+}$ and protons are strongly coupled to maintain acidic $\mathrm{pH}$ in lysosomes. ${ }^{50}$ Therefore, we examined the effects of SsD treatment on the lysosomal $\mathrm{Ca}^{2+}$ content by measuring the cytosolic $\mathrm{Ca}^{2+}$ changes elicited by Glycyl-I-phenylalanine 2-naphthylamide (GPN), which specifically breaks the lysosomal membrane ${ }^{51}$ to release lysosomal $\mathrm{Ca}^{2+} .52 \mathrm{As}$ shown in Fig. 4c, GPN induced similar levels of cytosolic $\mathrm{Ca}^{2+}$ increases in the presence or absence of $\mathrm{SsD}$ pretreatment, indicating that SsD does not change lysosomal $\mathrm{Ca}^{2+}$ content. Taken together, these data suggest that SsD-mediated autophagy inhibition is independent of ER or lysosomal $\mathrm{Ca}^{2+}$ changes.

RAB5 is required for autophagy inhibition by SsD

Growing evidence indicates that RAB small GTPases play important roles in autophagy regulation, ${ }^{53}$ and thus, we assessed 
A

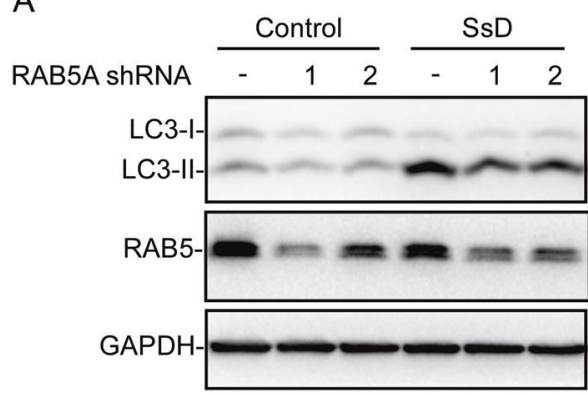

B

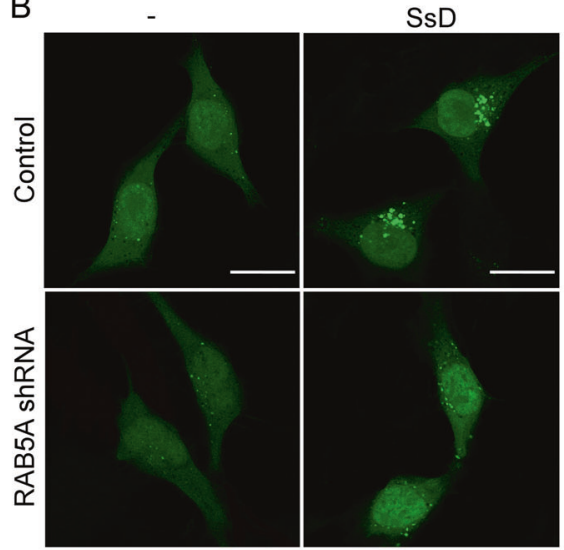

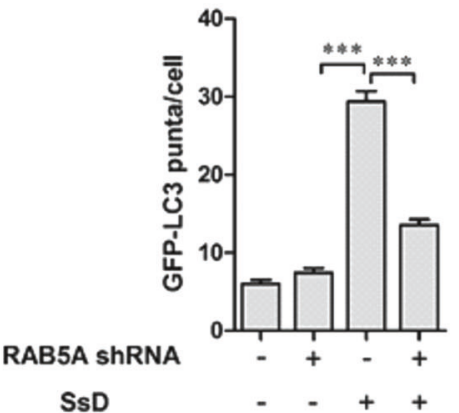

SsD

C Control

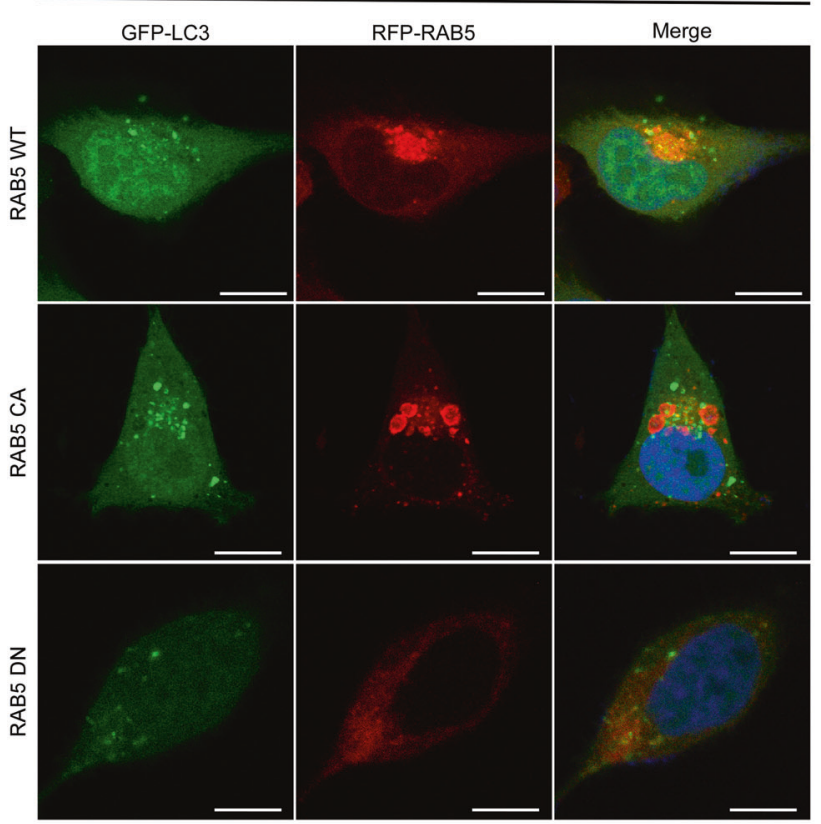

$\mathrm{SsD}$
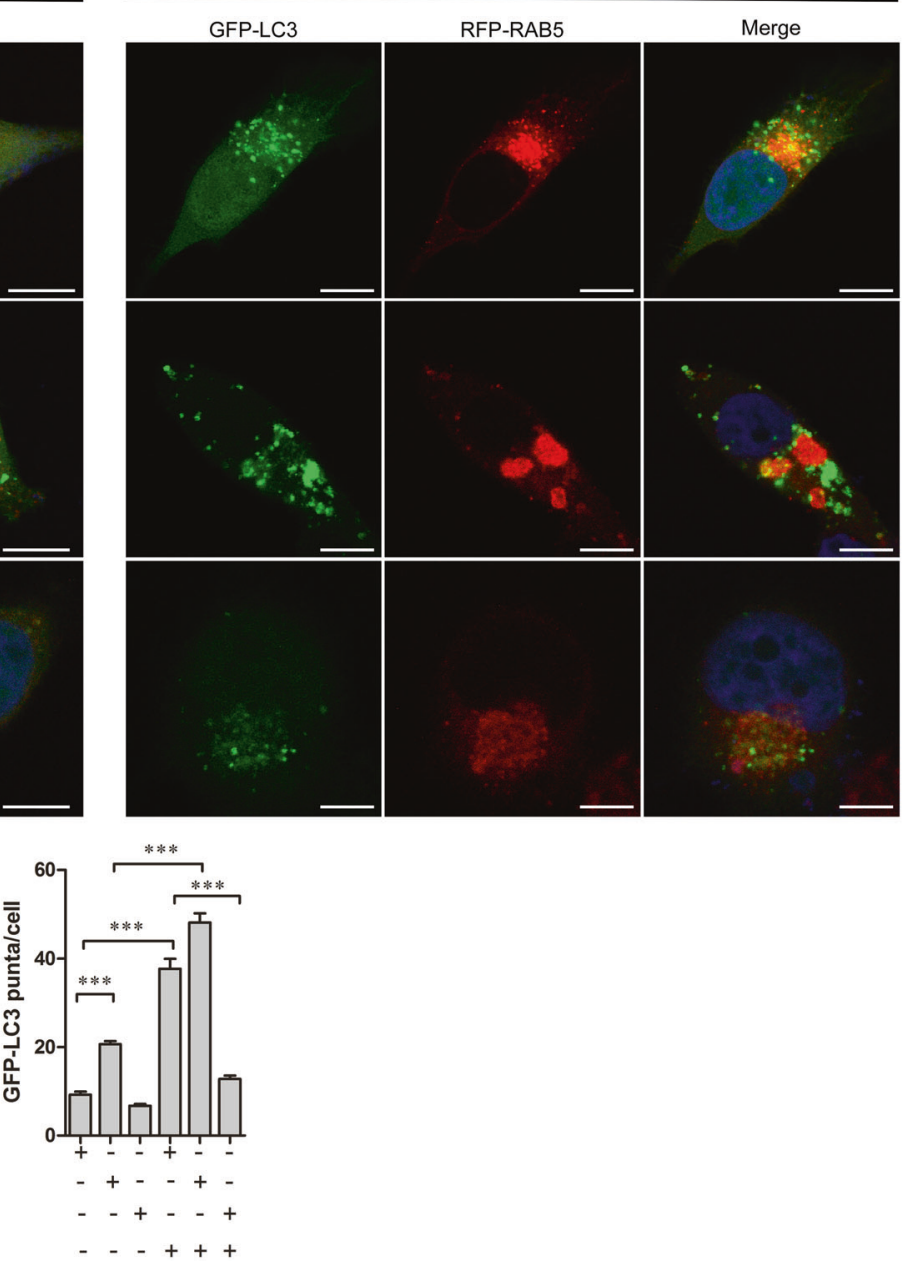

Fig. 5 RAB5 is required for autophagy inhibition by SsD. a RAB5A knockdown inhibited SsD (15 $\mu$ M)-induced accumulation of LC3-II in HeLa cells. b RAB5A knockdown inhibited SsD $(15 \mu \mathrm{M})$-induced accumulation of GFP-LC3-Il puncta in HeLa cells. Scale bar $=10 \mu \mathrm{m}$. Quantification of GFP-LC3-II puncta per cell is presented as the mean \pm S.E., $n=\sim 50$ cells of 3 independent experiments. The asterisk $\left(^{*}\right)$ symbols indicate $p<0.05$ by $t$ test analysis. c Overexpression of a constitutively active form of RAB5A (RAB5A-CA) enhanced the inhibitory effect of SsD, whereas the overexpression of a dominant-negative form of RAB5A (RAB5A-DN) prevented the increase of GFP-LC3 puncta induced by SsD. Scale bar $=10 \mu \mathrm{m}$. Quantification of GFP-LC3-II puncta per cell is presented as the mean \pm S.E., $n=\sim 50$ cells of 3 independent experiments

the role of $\mathrm{RAB}$ small GTPase in SsD-mediated autophagy inhibition. RAB7 knockdown in HeLa cells induced the accumulation of LC3-II, but SsD, SsA, or BAF treatment of RAB7-knockdown cells failed to further increase LC3-II levels, again suggesting that SsD and SsA, similarly to BAF, inhibit the autophagosomallysosomal fusion (Fig. S4). In contrast, the knockdown of RAB5A, a small GTPase known to be involved in early endocytosis, ${ }^{54}$ markedly reversed the SsD-induced accumulation of LC3-II compared to control HeLa cells (Fig. 5a). Similarly, the SsDinduced accumulation of GFP-LC3 puncta was significantly inhibited in RAB5A knockdown cells compared to control cells (Fig. 5b). Likewise, the overexpression of a dominant-negative 
A

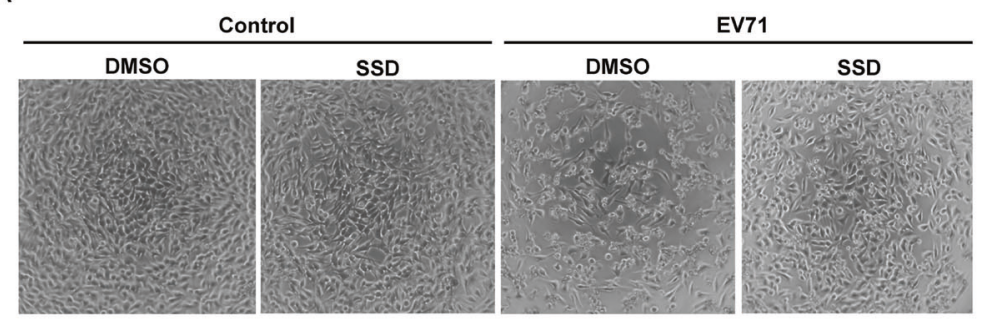

B

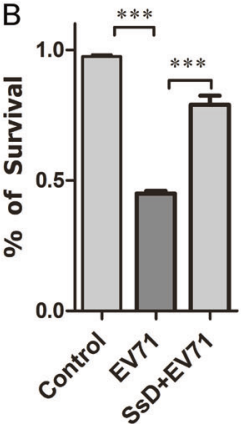

C
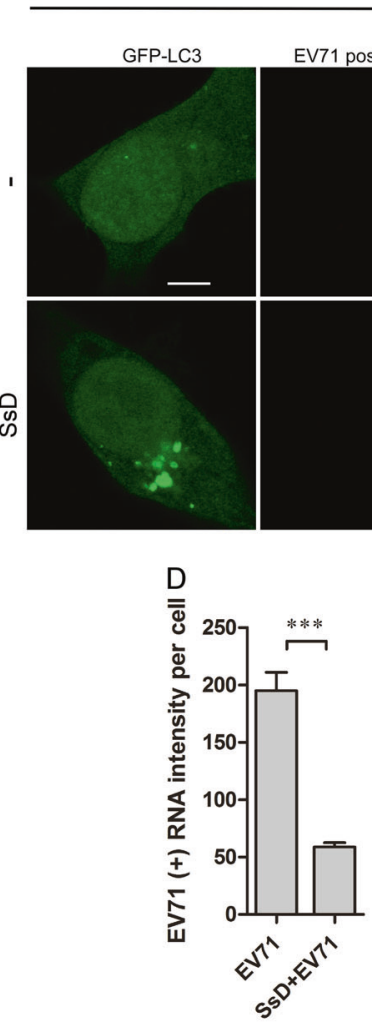

$F$

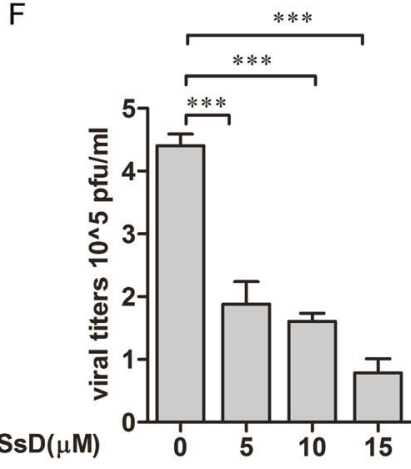

E

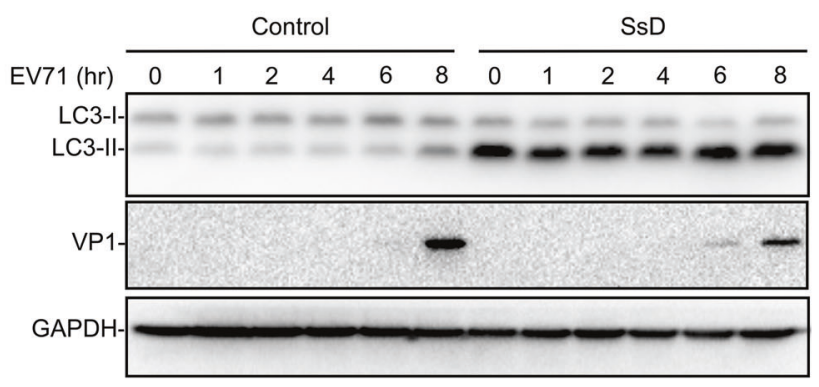

G
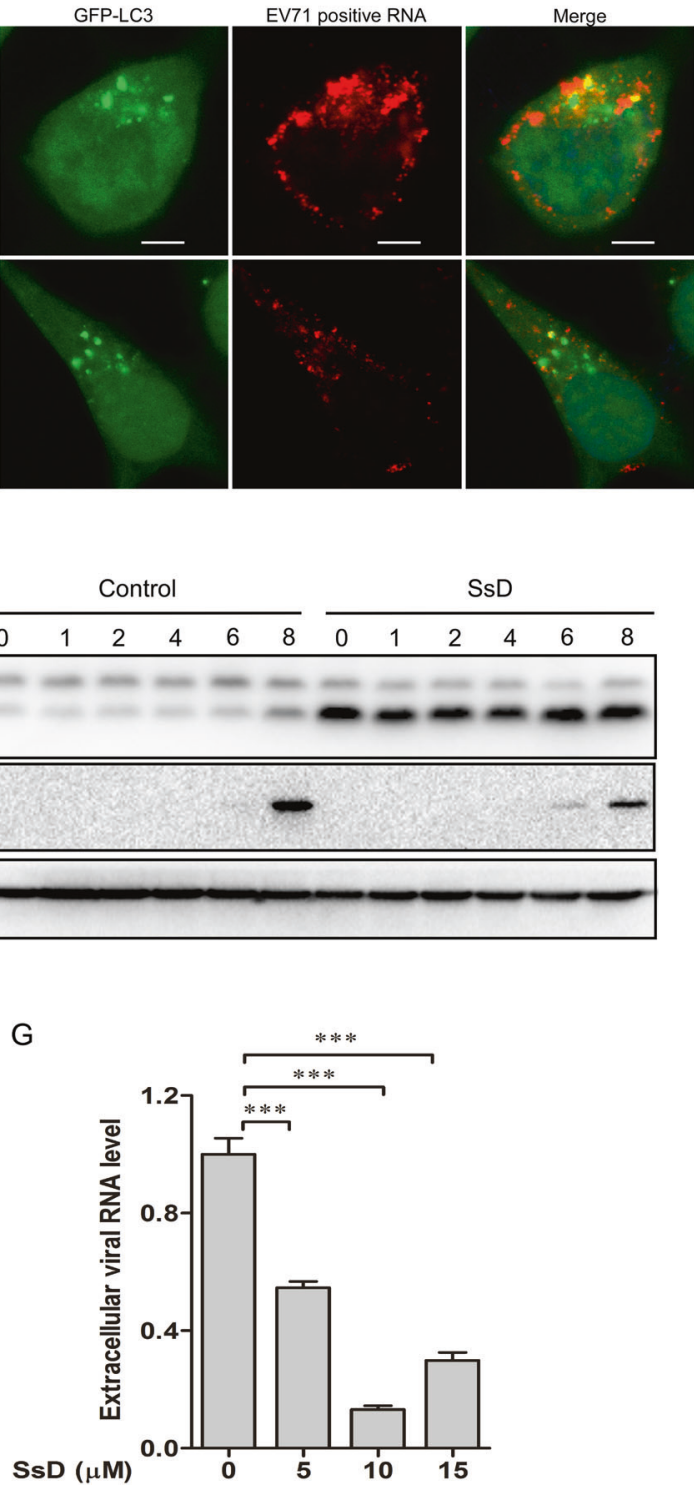

summary, these data clearly demonstrated that RAB5 is required for autophagy inhibition by SsD or SsA.

SsD inhibits EV-A71 infection in HeLa cells

It has been shown that EV-A71 induces autophagy in host cells, which might in turn facilitate the synthesis of viral RNA and proteins. $^{29-33}$ Therefore, we reasoned that SsD could inhibit autophagy to suppress EV-A71 infection. As expected, upon 
Fig. 6 SsD inhibits EV-A71 replication in HeLa cells. a HeLa cells pretreated with or without SsD $(15 \mu M)$ were infected with EV-A71 (MOI = 1) for $12 \mathrm{~h}$. SsD pretreatment greatly reduced the number of cytopathic cells after EV-A71 infection. b HeLa cells were infected with EV-A71 $(\mathrm{MOI}=1)$ in the presence or absence of SsD $(15 \mu \mathrm{M})$ for $12 \mathrm{~h}$, and MTT assays were performed. The graph represents data from three independent experiments, and data are expressed as the mean \pm S.D., $n=3$. The asterisk ${ }^{*}$ ) symbols indicate $p<0.05$ by $t$ test analysis. c and d GFP-LC3-expressing HeLa cells were infected with EV-A71 $(\mathrm{MOI}=1)$ in the presence or absence of SsD $(15 \mu \mathrm{M})$ for $5 \mathrm{~h}$, and EV-A71 positive strand RNA hybridization was performed (c). Quantification of EV-A71 positive RNA intensity per cell is expressed as the mean \pm S.E., $n=\sim 50$ cells from 3 independent experiments. The asterisk (*) symbols indicate $P<0.05$ by Student's $t$ Test analysis (d). e HeLa cells were infected with EV-A71 $(\mathrm{MOI}=1)$ in the presence or absence of SsD $(15 \mu \mathrm{M})$ for the indicated times, followed by LC3, VP1, and GAPDH immunoblot analyses. f Viral titers were significantly decreased by SsD treatment in a dose-dependent manner after EV-A71 infection. WT HeLa cells were infected with EV-A71 $(\mathrm{MOI}=1)$ for $12 \mathrm{~h}$. g SSD $(15 \mu \mathrm{M})$ treatment markedly reduced extracellular EV-A71 RNA level in a dose-dependent manner. WT HeLa cells were infected with EV-A71 $(\mathrm{MOI}=1)$ for $12 \mathrm{~h}$

EV-A71 $(\mathrm{MOI}=1)$ infection, many cells developed cytopathic effects, showing a rounded shape or even detaching from the culture well. Treatment with SsD during EV-A71 infection greatly decreased the number of cytopathic cells, whereas SsD $(15 \mu \mathrm{M})$ alone showed little toxicity in HeLa cells (Fig. 6a). To better quantify the protective effect of SsD against EV-A71 infection, an MTT cell viability assay was performed. As expected, SsD significantly inhibited EV-A71-induced HeLa cell death (Fig. 6b).

To determine whether the anti-EV-A71 function of SSD results from its inhibition of the late stage of autophagy, we detected the EV-A71 positive RNA strand by RNA in situ hybridization. As shown in Fig. 6c, d, the EV-A71 positive RNA strand was significantly reduced by SsD treatment compared to the level in EV-A71infected cells without SsD treatment, indicating that the replication of EV-A71 viral RNA was inhibited by SsD treatment. Likewise, the expression level of VP1 was significantly decreased by SsD treatment during EV-A71 infection (Fig. 6e). To further detect the antiviral activity of $\mathrm{SsD}$, we measured the viral titers in the culture medium. As shown in Fig. $6 \mathrm{f}$, viral titers were significantly decreased by treatment with $\mathrm{SsD}$ in a dose-dependent manner. In addition, we detected secreted EV-A71 virions in the culture medium by measuring extracellular EV-A71 RNA. Treatment with SsD markedly reduced the extracellular EV-A71 RNA level during EV-A71 infection (Fig. 6g).

To further assess the role of autophagy in EV-A71 infection of cells, we infected HeLa cells with EV-A71 in the presence of BAF, a known autophagosome-lysosome fusion inhibitor similar to SsD. As expected, the treatment of HeLa cells with BAF dosedependently inhibited the expression of VP1 induced by EV-A71 infection (Fig. 7a). Likewise, the knockdown of ATG5, an important protein for early autophagy progression, markedly inhibited the expression of VP1 after EV-A71 infection in HeLa cells (Fig. 7b); however, the SsD treatment of ATG5-knockdown HeLa cells failed to further inhibit VP1 expression after EV-A71 infection (Fig. 7c). In contrast, we found that treatment of HeLa cells with rapamycin, an mTOR inhibitor known to induce autophagy, markedly increased the levels of VP1 protein (Fig. 7d). Interestingly, the SsD treatment of cells markedly inhibited VP1 levels induced by EV-A71 infection in the presence or absence of Torin-1, another mTOR inhibitor (Fig. 7e). Taken together, these data suggested that the ability of SsD to inhibit the late stage of autophagy contributes to its antiEV-A71 effects

\section{DISCUSSION}

Here we found that the treatment of cells with SsD or SsA, active components in Bupleurum falcatum, inhibited autophagosomelysosome fusion and resulted in the accumulation of autophagosomes (Figs. 1 and 2). We also found that SsD or SsA treatment increased lysosomal $\mathrm{pH}$ and induced the nuclear localization of TFEB (Fig. 3) but did not affect ER or lysosome $\mathrm{Ca}^{2+}$ pools (Fig. 4). Moreover, we found that RAB5 was required for SsD-induced or SsA-induced autophagosomal-lysosomal fusion blockage (Fig. 5), but $\mathrm{SsD}$ and $\mathrm{SsA}$ failed to further inhibit autophagosomallysosomal fusion in RAB7 knockdown cells (Fig. S4). Interestingly,
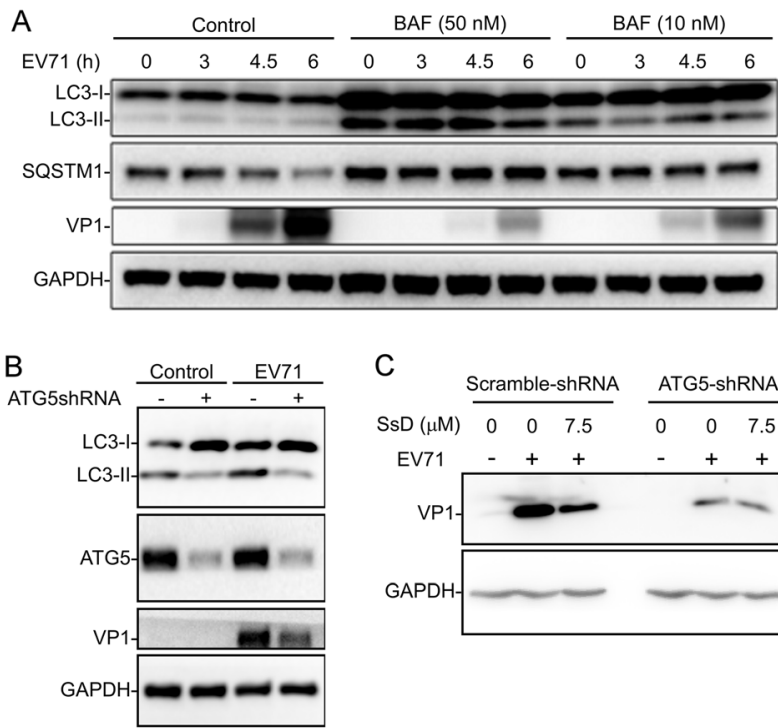

C
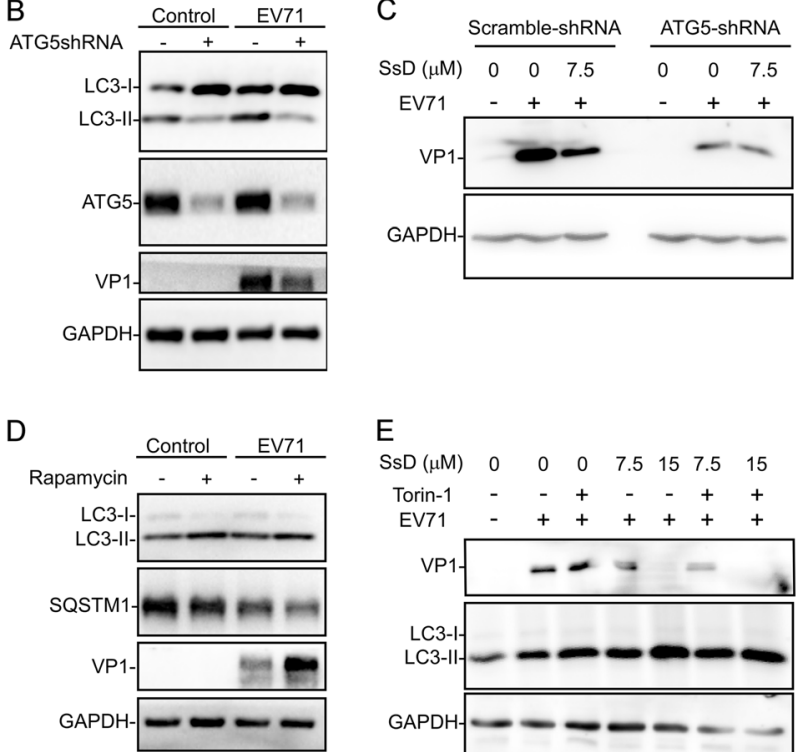

E

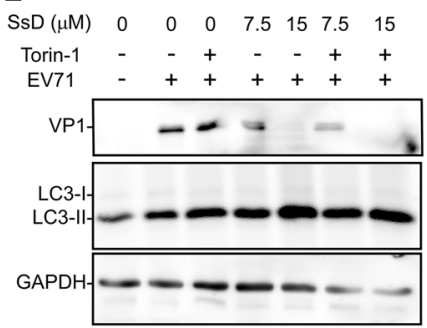

Fig. 7 Autophagy is involved in EV-A71 replication in HeLa cells. a VP1 synthesis was dramatically reduced by BAF (50 or $10 \mathrm{nM}$ ) treatment in EV71-infected cells compared to cells without BAF treatment. b ATG5 knockdown markedly inhibited EV-A71 infection in HeLa cells. c SsD treatment failed to further inhibit AV-A71 infection in ATG5-knockdown HeLa cells. d Rapamycin treatment enhanced EV-A71 infection in HeLa cells. e SsD treatment markedly inhibited EV-A71 infection in HeLa cells treated with or without Torin-1

we found that SsD strongly reduced EV-A71 RNA replication, viral protein synthesis, and virus titers in host cells (Fig. 6a-g), and this inhibition was correlated with inhibitory effects on autophagy. Likewise, autophagy inhibition by ATG5 knockdown or BAF treatment suppressed EV-A71 viral replication (Fig. 7a, b), whereas autophagy induction by rapamycin or Torin-1 promoted replication (Fig. 7d, e). Thus, we speculated that autophagy inhibition by SsD or SsA acts as a protective mechanism against EV-A71 infection.

Previous research showed that SsD treatment of cells increases LC3-II levels and LC3-II puncta, but the authors concluded that SsD induced earlier autophagy, which ultimately resulted in 
autophagic cell death. ${ }^{56}$ Here, we found that the SsD-induced LC3II puncta failed to co-localize with LAMP1 or RAB7, two lysosomal markers (Fig. 2c, i), but exhibited strong co-localization with STX17, an autophagosomal marker (Fig. 2e). Moreover, SsD failed to further increase LC3-II levels in BAF-treated or RAB7 knockdown cells (Fig. $2 \mathrm{~b}$ and S4). Taken together, these data clearly indicate that SsD and SsA inhibit the autophagosome-lysosome fusion, and the increased levels of LC3-II and p62 and accumulated LC3-II puncta in SsD-treated cells are due to the blockage of autophagosomal-lysosomal fusion rather than autophagy induction at earlier stages.

We also found that RAB5A was required for autophagy arrest by SsD or SsA (Fig. 5 and S5). We speculate that SsD and SsA might indirectly activate RAB5, which subsequently contributes to the defects in lysosome biogenesis and results in lysosomal $\mathrm{pH}$ elevation. RAB5 is a small GTPase. GTP-bound RAB5 is the active form, whereas GDP-bound RAB5 is the inactive form. A guanine nucleotide exchange factor (GEF) is responsible for the conversion from GTP-bound RAB5 to GDP-bound RAB5. Both the GTPaseactivating protein (GAP) and GTPase activity of RAB5 are involved in the switch from RAB5-GTP to RAB5-GDP. Many GEFs and GAPs of RAB5 have been discovered. ${ }^{57}$ There are several possibilities for RAB5A activation by SsD. One is that SsD directly inhibits RAB5 GTPase activity; another is that a RAB5 GEF is activated by SsD or a RAB5 GAP is inhibited by SsD, thereby activating RAB5 as a RAB5GTP form. Notably, we recently found that the small chemical vacuolin-1 also indirectly activates RAB5 to arrest autophagosomelysosome fusion. ${ }^{37}$

To investigate the role of autophagy in EV71 infection, HeLa cells were selected in this study to undergo EV71 infection. HeLa cells are susceptible to EV-A71 infection, enabling efficient progeny viral propagation. The viral growth kinetics and the cytopathic effects of EV-A71 in HeLa cells are similar to those of other popularly used cell lines to study EV-A71. HeLa cells have been used to study enteroviruses, including EV-A71. ${ }^{58-60}$ In addition, HeLa cells are autophagy competent. ${ }^{37,61}$ However, it is worth noting that HeLa cells are less susceptible to EV-A71 infection than Rhabdomyosarcoma (RD) cells or Vero cells, two popular cell lines used to study EV71, and thus, a higher titer of EVA71 virus was used to infect HeLa cells.

\section{ACKNOWLEDGEMENTS}

We thank members of the Yue lab for their advice on the preparation of this manuscript. This work was supported by Hong Kong Research Grant Council (RGC) grants (785213, 17126614, and 11101717), NSFC (21778045), the CAS-Croucher Funding Scheme, the Guangdong-Hong Kong joint innovation Research Scheme (2016A050503010), a Shenzhen government research grant (JCYJ20160229165235739 and JCYJ20170413141331470) to J.Y., and it was partly supported by GRF (14105214) and NSFC $(81671995,81471964)$ grants to M.L.H.

\section{AUTHOR CONTRIBUTIONS}

C.L., L.H., W.S., and Y.C. performed experiments, C.L., M.L.H., H.B., and J.Y. wrote the manuscript, and J.Y. conceived the study and designed the experiments.

\section{ADDITIONAL INFORMATION}

The online version of this article (https://doi.org/10.1038/s41392-019-0037-x) contains supplementary material, which is available to authorized users.

Competing interests: The authors declare no competing interests.

\section{REFERENCES}

1. Mizushima, N. \& Klionsky, D. J. Protein turnover via autophagy: implications for metabolism. Annu. Rev. Nutr. 27, 19-40 (2007).

2. Klionsky, D. J. The molecular machinery of autophagy: unanswered questions. J. Cell. Sci. 118, 7-18 (2005).
3. Levine, B. \& Klionsky, D. J. Development by self-digestion: molecular mechanisms and biological functions of autophagy. Dev. Cell. 6, 463-477 (2004).

4. Mizushima, N. Autophagy: process and function. Genes Dev. 21, 2861-2873 (2007).

5. Levine, B., Mizushima, N. \& Virgin, H. W. Autophagy in immunity and inflammation. Nature 469, 323-335 (2011).

6. Choi, A. M., Ryter, S. W. \& Levine, B. Autophagy in human health and disease. N. Engl. J. Med. 368, 1845-1846 (2013).

7. White, E. Deconvoluting the context-dependent role for autophagy in cancer. Nat. Rev. Cancer 12, 401-410 (2012).

8. Nixon, R. A. The role of autophagy in neurodegenerative disease. Nat. Med. 19, 983-997 (2013).

9. Galluzzi, L., Bravo-San Pedro, J. M., Levine, B., Green, D. R. \& Kroemer, G. Pharmacological modulation of autophagy: therapeutic potential and persisting obstacles. Nat. Rev. Drug Discov. 16, 487-511 (2017).

10. Gao, L., Jauregui, C. E. \& Teng, Y. Targeting autophagy as a strategy for drug discovery and therapeutic modulation. Future Med. Chem. 9, 335-345 (2017).

11. Rubinsztein, D. C., Codogno, P. \& Levine, B. Autophagy modulation as a potential therapeutic target for diverse diseases. Nat. Rev. Drug Discov. 11, 709-730 (2012).

12. Levy, J. M. M., Towers, C. G. \& Thorburn, A. Targeting autophagy in cancer. Nat. Rev. Cancer 17, 528-542 (2017).

13. Marinkovic, M., Sprung, M., Buljubasic, M. \& Novak, I. Autophagy modulation in cancer: current knowledge on action and therapy. Oxid. Med. Cell Longev. 2018, 8023821 (2018).

14. Morel, E. et al. Autophagy: a druggable process. Annu. Rev. Pharmacol. Toxicol. 57, 375-398 (2017).

15. Ashour, M. L. \& Wink, M. Genus Bupleurum: a review of its phytochemistry, pharmacology and modes of action. J. Pharm. Pharmacol. 63, 305-321 (2011).

16. Ushio, Y. \& Abe, H. Inactivation of measles virus and herpes simplex virus by saikosaponin d. Planta Med. 58, 171-173 (1992).

17. Li, X. Q., et al. Saikosaponins: a review of pharmacological effects. J Asian Nat. Prod. Res. 5, 1-13 (2018).

18. Wong, V. K., Zhou, H., Cheung, S. S., Li, T. \& Liu, L. Mechanistic study of saikosaponin-d (Ssd) on suppression of murine T lymphocyte activation. J. Cell. Biochem. 107, 303-315 (2009).

19. Wong, V. K. et al. Saikosaponin-d enhances the anticancer potency of tnf-alpha via overcoming its undesirable response of activating nf-kappa b signalling in cancer cells. Evid.-Based Complement. Altern. Med. 2013, 745295 (2013).

20. Tundis, R. et al. Cytotoxic activity and inhibitory effect on nitric oxide production of triterpene saponins from the roots of Physospermum verticillatum (Waldst \& Kit) (Apiaceae). Bioorg. Med. Chem. 17, $4542-4547$ (2009).

21. Lin, T. Y., Chu, C. \& Chiu, C. H. Lactoferrin inhibits enterovirus 71 infection of human embryonal rhabdomyosarcoma cells in vitro. J. Infect. Dis. 186, 1161-1164 (2002).

22. Ho, M. et al. An epidemic of enterovirus 71 infection in Taiwan. Taiwan Enterovirus Epidemic Working Group. N. Engl. J. Med. 341, 929-935 (1999).

23. Schmidt, N. J., Lennette, E. H. \& Ho, H. H. An apparently new enterovirus isolated from patients with disease of the central nervous system. J. Infect. Dis. 129, 304-309 (1974).

24. Chumakov, M. et al. Enterovirus 71 isolated from cases of epidemic poliomyelitislike disease in Bulgaria. Arch. Virol. 60, 329-340 (1979).

25. Melnick, J. L. Enterovirus type 71 infections: a varied clinical pattern sometimes mimicking paralytic poliomyelitis. Rev. Infect. Dis. 6(Suppl 2), S387-S390 (1984).

26. Chan, L. G. et al. Deaths of children during an outbreak of hand, foot, and mouth disease in sarawak, malaysia: clinical and pathological characteristics of the disease. For the outbreak study group. Clin. Infect. Dis. 31, 678-683 (2000).

27. Ooi, M. H., Wong, S. C., Lewthwaite, P., Cardosa, M. J. \& Solomon, T. Clinical features, diagnosis, and management of enterovirus 71. Lancet Neurol. 9, 1097-1105 (2010).

28. Lei, X. et al. Cleavage of interferon regulatory factor 7 by enterovirus $713 C$ suppresses cellular responses. J. Virol. 87, 1690-1698 (2013).

29. Huang, S. C., Chang, C. L., Wang, P. S., Tsai, Y. \& Liu, H. S. Enterovirus 71-induced autophagy detected in vitro and in vivo promotes viral replication. J. Med. Virol. 81, 1241-1252 (2009).

30. Lee, Y. R., Wang, P. S., Wang, J. R. \& Liu, H. S. Enterovirus 71-induced autophagy increases viral replication and pathogenesis in a suckling mouse model. J. Biomed. Sci. 21, 80 (2014).

31. Wang, $\mathrm{H}$. et al. Berberine inhibits enterovirus 71 replication by downregulating the MEK/ERK signaling pathway and autophagy. Virol. J. 14, 2 (2017).

32. Fu, Y. et al. Enterovirus 71 induces autophagy by regulating has-miR-30a expression to promote viral replication. Antivir. Res. 124, 43-53 (2015).

33. $\mathrm{Xi}, \mathrm{X}$. et al. The interplays between autophagy and apoptosis induced by enterovirus 71. PLOS ONE 8, e56966 (2013).

34. Lu, J. et al. Viral kinetics of Enterovirus 71 in human abdomyosarcoma cells. World J. Gastroenterol. 17, 4135-4142 (2011). 
35. Lu, J. et al. Enterovirus 71 disrupts interferon signaling by reducing the level of interferon receptor 1. J. Virol. 86, 3767-3776 (2012).

36. Yi, L., He, Y., Chen, Y., Kung, H. F. \& He, M. L. Potent inhibition of human enterovirus 71 replication by type I interferon subtypes. Antivir. Ther. 16, 51-58 (2011).

37. Lu, Y. et al. Vacuolin-1 potently and reversibly inhibits autophagosome-lysosome fusion by activating RAB5A. Autophagy 10, 1895-1905 (2014).

38. Bankers-Fulbright, J. L., Kephart, G. M., Bartemes, K. R., Kita, H. \& O'Grady, S. M. Platelet-activating factor stimulates cytoplasmic alkalinization and granule acidification in human eosinophils. J. Cell Sci. 117, 5749-5757 (2004).

39. Muir, P. et al. Molecular typing of enteroviruses: current status and future requirements. The European Union Concerted Action on Virus Meningitis and Encephalitis. Clin. Microbiol. Rev. 11, 202-227 (1998).

40. Kawai, A., Uchiyama, H., Takano, S., Nakamura, N. \& Ohkuma, S. Autophagosomelysosome fusion depends on the $\mathrm{pH}$ in acidic compartments in $\mathrm{CHO}$ cells. Autophagy 3, 154-157 (2007).

41. Davis-Kaplan, S. R., Ward, D. M., Shiflett, S. L. \& Kaplan, J. Genome-wide analysis of iron-dependent growth reveals a novel yeast gene required for vacuolar acidification. J. Biol. Chem. 279, 4322-4329 (2004).

42. DePedro, H. M. \& Urayama, P. Using LysoSensor Yellow/Blue DND-160 to sense acidic pH under high hydrostatic pressures. Anal. Biochem. 384, 359-361 (2009).

43. Roczniak-Ferguson, A. et al. The transcription factor TFEB links mTORC1 signaling to transcriptional control of lysosome homeostasis. Sci. Signal. 5, ra42 (2012).

44. Settembre, C. et al. A lysosome-to-nucleus signalling mechanism senses and regulates the lysosome via mTOR and TFEB. EMBO J. 31, 1095-1108 (2012).

45. Settembre, C. et al. TFEB links autophagy to lysosomal biogenesis. Science $\mathbf{3 3 2}$, 1429-1433 (2011)

46. Sun, F., Xu, X., Wang, X. \& Zhang, B. Regulation of autophagy by Ca2. Tumour Biol. 37, 15467-15476 (2016).

47. Smith, S. M., Renden, R. \& von Gersdorff, H. Synaptic vesicle endocytosis: fast and slow modes of membrane retrieval. Trends Neurosci. 31, 559-568 (2008).

48. Sudhof, T. C. The synaptic vesicle cycle. Annu. Rev. Neurosci. 27, 509-547 (2004).

49. Jahn, R. \& Fasshauer, D. Molecular machines governing exocytosis of synaptic vesicles. Nature 490, 201-207 (2012).

50. Morgan, A. J., Platt, F. M., Lloyd-Evans, E. \& Galione, A. Molecular mechanisms of endolysosomal Ca2+ signalling in health and disease. Biochem. J. 439, 349-374 (2011).

51. Berg, T. O., Stromhaug, E., Lovdal, T., Seglen, O. \& Berg, T. Use of glycyl-Lphenylalanine 2-naphthylamide, a lysosome-disrupting cathepsin C substrate, to distinguish between lysosomes and prelysosomal endocytic vacuoles. Biochem. J. 300(Pt 1), 229-236 (1994).

52. Yeung-Yam-Wah, V., Lee, A. K. \& Tse, A. Arachidonic acid mobilizes Ca2+ from the endoplasmic reticulum and an acidic store in rat pancreatic beta cells. Cell Calcium 51, 140-148 (2012).

53. Szatmari, Z. \& Sass, M. The autophagic roles of Rab small GTPases and their upstream regulators: a review. Autophagy 10, 1154-1166 (2014).

54. Rink, J., Ghigo, E., Kalaidzidis, Y. \& Zerial, M. Rab conversion as a mechanism of progression from early to late endosomes. Cell 122, 735-749 (2005).

55. Bohdanowicz, M., Balkin, D. M., De Camilli, P. \& Grinstein, S. Recruitment of OCRL and Inpp5B to phagosomes by Rab5 and APPL1 depletes phosphoinositides and attenuates Akt signaling. Mol. Biol. Cell. 23, 176-187 (2012).

56. Wong, V. K. et al. Saikosaponin-d, a novel SERCA inhibitor, induces autophagic cell death in apoptosis-defective cells. Cell Death Dis. 4, e720 (2013).

57. Stenmark, H. Rab GTPases as coordinators of vesicle traffic. Nat. Rev. Mol. Cell Biol. 10, 513-525 (2009).

58. Tan, C. W., Chan, Y. F., Sim, K. M., Tan, E. L. \& Poh, C. L. Inhibition of enterovirus 71 (EV-71) infections by a novel antiviral peptide derived from EV-71 capsid protein VP1. PLoS One 7, e34589 (2012).

59. Falah, N. et al. Blocking human enterovirus 71 replication by targeting viral $2 \mathrm{~A}$ protease. J. Antimicrob. Chemother. 67, 2865-2869 (2012).

60. Du, N. et al. Cell surface vimentin is an attachment receptor for enterovirus 71. J. Virol. 88, 5816-5833 (2014).

61. Wang, Q. et al. Mechanistic study of TRPM2-Ca(2+)-CAMK2-BECN1 signaling in oxidative stress-induced autophagy inhibition. Autophagy 12, 1340-1354 (2016).

Open Access This article is licensed under a Creative Commons Attribution 4.0 International License, which permits use, sharing, adaptation, distribution and reproduction in any medium or format, as long as you give appropriate credit to the original author(s) and the source, provide a link to the Creative Commons license, and indicate if changes were made. The images or other third party material in this article are included in the article's Creative Commons license, unless indicated otherwise in a credit line to the material. If material is not included in the article's Creative Commons license and your intended use is not permitted by statutory regulation or exceeds the permitted use, you will need to obtain permission directly from the copyright holder. To view a copy of this license, visit http://creativecommons. org/licenses/by/4.0/.

(c) The Author(s) 2019 